\title{
Total sleep time, alcohol consumption, and the duration and severity of alcohol hangover
}

\section{Marith van Schrojenstein Lantman' Marlou Mackus' \\ Thomas Roth ${ }^{2}$ \\ Joris C Verster ${ }^{1,3,4}$}

'Utrecht Institute for Pharmaceutical Sciences, Division of Pharmacology, Utrecht University, Utrecht, The Netherlands; ${ }^{2}$ Sleep Disorders and Research Center, Henry Ford Health System, Detroit, Michigan, USA; ${ }^{3}$ Institute for Risk Assessment Sciences (IRAS), Utrecht University, Utrecht, The Netherlands; ${ }^{4}$ Centre for Human Psychopharmacology, Swinburne University, Melbourne VIC, Australia
Correspondence: Joris C Verster Utrecht Institute for Pharmaceutical Sciences, Division of Pharmacology, Utrecht University, Universiteitsweg 99, 3584 CG, Utrecht, The Netherlands $\mathrm{Tel}+3 \mid 302536909$

Fax +3I 302537900

Email j.c.verster@uu.nl
This article was published in the following Dove Press journal:

Nature and Science of Sleep

29 June 2017

Number of times this article has been viewed

Introduction: An evening of alcohol consumption often occurs at the expense of sleep time. The aim of this study was to determine the relationship between total sleep time and the duration and severity of the alcohol hangover.

Methods: A survey was conducted among Dutch University students to collect data on their latest alcohol hangover. Data on alcohol consumption, total sleep time, hangover severity, and duration were collected. Alcohol consumption and hangover severity and duration were compared for participants who (a) slept $<5$ hours, (b) slept between 5 and 7 hours, or (c) slept $>7$ hours.

Results: Data from N=578 students ( $40.1 \%$ men and $59.9 \%$ women) were included in the statistical analyses. Significant correlations were found between total sleep time and alcohol consumption ( $r=0.117, p=0.005)$, hangover severity $(r=-0.178, p=0.0001)$ and hangover duration ( $r=0.168, p=0.0001$ ). In contrast, total alcohol consumption did not correlate significantly with overall hangover severity or duration. Those who slept longer than 7 hours consumed significantly more alcohol $(p=0.016)$ and reported extended hangover duration $(p=0.004)$. However, they also reported significantly less severe hangovers $(p=0.001)$ than students who slept $<7$ hours.

Conclusion: Reduced total sleep time is associated with more severe alcohol hangovers.

Keywords: alcohol, hangover, duration, severity, total sleep time

\section{Introduction}

Due to next-day commitments, an evening of alcohol consumption often occurs at the expense of total sleep time. The most frequently reported consequence of heavy alcohol consumption is the alcohol hangover. The alcohol hangover refers to the combination of mental and physical symptoms, experienced the day after a single episode of heavy drinking, starting when blood alcohol concentration approaches zero. ${ }^{1}$

Only a few studies have investigated the relationship between heavy alcohol consumption and subsequent sleep, and impact on next day functioning and mood. Finnigan et al examined the next-day effects of consuming alcohol (blood alcohol concentration [BAC] $0.10 \%$ ) on psychomotor performance, subjective state, and quality of sleep. ${ }^{2}$ In this double-blind placebo-controlled study of 40 men who were moderate-to-heavy social drinkers, no significant effects were found on psychomotor performance. However, after consuming alcohol subjects fell asleep faster, and reported reduced alertness during the hangover state. McKinney and Coyle examined mood and anxiety in 48 social drinkers the day after heavy drinking, and on an alcohol-free control day. ${ }^{3}$ Applying a naturalistic study design, subjects consumed the amount, type of alcohol at their own pace and venue of choice, without the presence of the investigators. However, they were 
asked to limit drinking to between $10 \mathrm{pm}$ and $2 \mathrm{am}$, but there were no restrictions placed on the subjects, sleep behavior. Heavy alcohol consumption had a negative impact on nextday mood including increased anxiety levels. Disrupted sleep was reported after alcohol consumption and fatigue was significantly increased during the hangover state. These data illustrate that drinking goes at the expense of sleep. In the alcohol condition subjects went to bed significantly later when compared to the alcohol-free day, resulting in a significantly reduced total sleep time. The higher the intake of alcohol, the shorter the corresponding sleep latency. After alcohol, subjects qualified their sleep as less satisfying, refreshing, or restful. Rohsenow et al investigated the impact of heavy drinking on hangover, perceived sleep, and nextday ship power plant operation. ${ }^{4}$ Maritime academy cadets $(n=61)$ consumed alcohol to achieve a BAC of $0.115 \%$. Next morning, 8 hours thereafter, they were tested. No significant difference relative to the alcohol-free test day was observed in performance on the diesel power plant simulator. While total sleep time was not affected by alcohol consumption, subjects reported significantly improved sleep quality and reduced sleep latency after alcohol. In a subsequent study, Rohsenow et al again examined the effects of heavy alcohol consumption on sleep and neurocognitive functioning. ${ }^{5}$ This study further examined whether drinks with low and high congener content (vodka versus bourbon, respectively) had a differential impact on sleep, and next-day hangover severity and performance outcomes. Young, heavy, social drinkers $(n=95)$ participated in this double-blind study. After consuming alcohol to achieve a BAC of $0.11 \%$, subjects went to bed. Sleep was monitored using polysomnography. During hangover, neurocognitive performance showed significant impairment on tests combining sustained attention and rapid responding. Hangover severity correlated with poorer performance. Polysomnographic assessments revealed that alcohol consumption decreased sleep efficiency and rapid eye movement sleep, and increased wake time and next-day self-reported sleepiness. Hangover severity was significantly worse in subjects with reduced sleep efficiency, and subjects with higher hangover severity scores had a reduced total sleep time and spent less time in rapid eye movement sleep. No significant difference on sleep or performance outcomes was observed for drinks with low or high congener content. However, hangover severity was significantly worse after consuming bourbon. No significant gender differences were observed.

Together, the findings from these studies support the hypothesis that the interrelationship between heavy alcohol consumption and subsequent sleep quality and duration may have a significant impact on next day hangover severity, performance impairment, and mood disturbances. Therefore, the aim of the current analyses was to further examine the relationship between total sleep time and sleep quality, and the duration and severity of the alcohol hangover. To determine this, data from Penning et al was analyzed from a survey on alcohol hangover. ${ }^{6}$

\section{Methods}

Dutch students were approached at different locations on the campus of Utrecht University, and asked to complete a retrospective survey. Written informed consent was obtained from all participants; no formal ethics approval was required to conduct this type of survey research, according to the Central Committee on Research Involving Human Subjects (CCMO). There was no participation rate recorded, but the vast-majority of students were willing to complete the survey.

Data were collected on alcohol consumption during their latest heavy drinking session, subsequent total sleep time, and next day hangover severity. Overall hangover severity was reported at every 2 hours after waking up (starting 4 am until midnight). At each time point, participants could rate their hangover severity on a scale ranging from 0 (absent) to 10 (extreme). The area under the curve was computed to reflect overall hangover severity, ranging from 0 (absent) to 111 (extreme). In addition, the severity of 47 individual hangover symptoms was scored on a scale ranging from 0 (absent) to 10 (extreme). ${ }^{6}$

The number of alcoholic drinks consumed was recorded, as was the start and stop time of drinking. This information, together with data on body weight and gender, was used to compute the estimated peak BAC (eBAC), using the modified Widmark formula. ${ }^{7}$ Finally, start and stop time of sleep was recorded to calculate total sleep time (TST).

Statistical analyses were conducted with SPSS, version 24 (IBM Corp., Armonk, NY, USA). Alcohol consumption, hangover severity, and duration were compared for participants who (1) slept $<5$ hours, (2) slept between 5 and 7 hours, or (3) slept $>7$ hours. Subjects were included in the analyses if they were aged 18-30 years and reported having a hangover after consuming alcohol during the past month.

\section{Results}

Data from 578 students (40.1\% men and 59.9\% women) were included in the statistical analyses. Their mean (SD) age was 20.0 (2.1) years and they consumed 20.5 (17.3) alcoholic drinks weekly. On average, they experience 2.7 (2.4) hangover days per month. On their latest heavy drinking occasions, they consumed 8.3 (5.8) alcoholic drinks to achieve a mean (SD) 
eBAC of $0.10(0.1) \%$. The average duration of hangover since their last drink was 18.4 (3.9) hours. The drinking session was followed on average by $6.5(2.1)$ hours of sleep. Table 1 summarizes the demographic data of the sample.

TST correlated significantly with hangover severity ( $r=0.178, p=0.0001)$ and duration $(r=0.168, p=0.0001)$, total alcohol consumption $(r=0.117, p=0.005)$, and eBAC ( $r=0.123, p=0.003)$. As expected, eBAC correlated significantly with alcohol consumed on the heavy drinking occasion ( $r=0.806, p=0.0001)$ and weekly alcohol consumption ( $r=0.302, p=0.0001)$. Weekly alcohol consumption also significantly related to the number of alcoholic drinks consumed on the latest heavy drinking occasion that resulted in a hangover $(r=0.530, p=0.0001)$. However, eBAC did not significantly correlate with hangover severity $(r=0.018$, $p=0.664)$ or duration $(r=-0.006, p=0.895)$. Similarly, total alcohol consumption also did not significantly correlate with overall hangover severity ( $p=0.953)$ and duration $(p=0.563)$.

The majority of students $(\mathrm{N}=313)$ slept between 5 and 7 hours. Those who slept longer than 7 hours consumed significantly more alcohol (9.3 versus 7.9 and 7.7 drinks, $p=0.016$ ), reported extended hangover duration (19.2 versus 18.3 and 17.5 hours, $p=0.004$ ), but reported significantly less severe hangovers (20.9 versus 23.9 and $27.5, p=0.001$ ) than students who slept 5-7 hours or $<5$ hours, respectively (see Table 1).

When compared to men, women consumed significantly less alcohol weekly (15.7 versus 27.7 drinks, $p=0.0001$ ), and significantly less alcohol on their latest heavy drinking episode (5.8 versus 12.0 drinks, $p=0.0001)$. Accordingly, eBAC was significantly lower in women when compared to men $(0.08 \%$ versus $0.13 \%, p=0.0001)$. Also, women slept significantly shorter hours than men $(6.2$ versus 6.9 hours, $p=0.0001$ ). However, the short TST, lower amounts of alcohol consumed, and eBAC were not reflected in significant differences between women and men in hangover duration (18.6 versus 18.1 hours, $p=0.167)$ and hangover severity $(24.5$ versus $22.2, p=0.056$ ).

\section{Individual symptoms}

TST correlated significantly with fatigue $(r=-0.166$, $p=0.0001)$, sleepiness $(r=-0.118, p=0.005)$, reduced appetite ( $r=-0.105, p=0.012)$, stomach pain $(r=-0.098, p=0.020)$, respiratory problems $(r=-0.84, p=0.045)$, and impulsivity $(r=-0.083, p=0.049)$. Individual symptom severity scores for each sleep group are summarized in Table 2.

Those with a TST over 7 hours reported significantly less severe scores on fatigue $(p=0.032)$ and feeling guilty $(p=0.038)$ when compared to those who slept 5 to 7 hours, and significantly less severe scores on fatigue $(p=0.0001)$, sleepiness $(p=0.004)$, and respiratory problems $(p=0.005)$

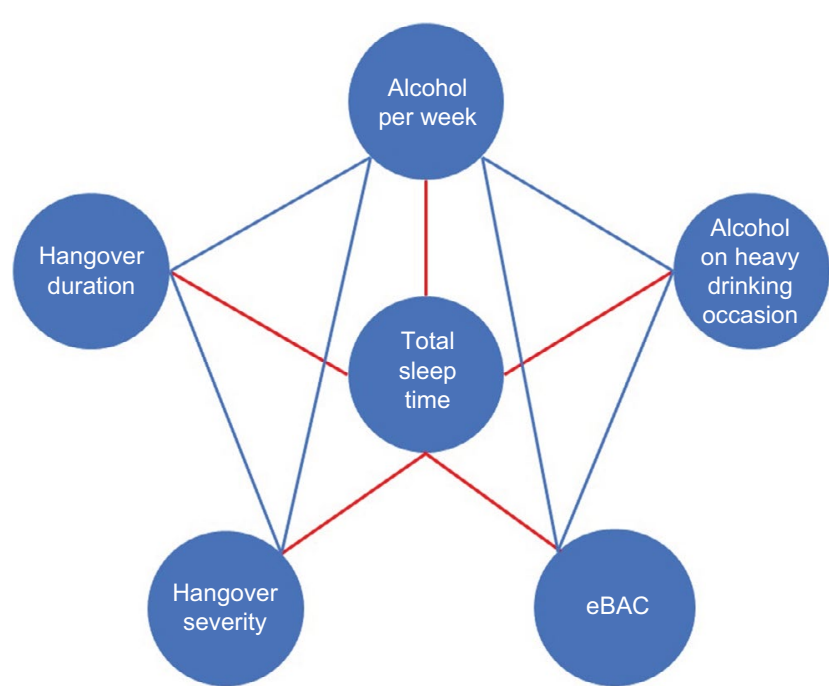

Figure I Associations of total sleep time with alcohol consumption and the severity and duration of the alcohol hangover.

Notes: Only significant associations $(p<0.05)$ are depicted. Red line indicates a negative correlation; blue line indicates a positive correlation.

Abbreviation: eBAC, estimated blood alcohol concentration.

Table I Demographics of subjects

\begin{tabular}{|c|c|c|c|c|}
\hline & Overall & $<5$ h sleep & 5-7 h sleep & $>7$ h sleep \\
\hline Number of subjects (\%) & $578(100)$ & $95(16.4)$ & $313(54.2)$ & $170(29.4)$ \\
\hline Age (years) & $20.0(2.1)$ & $19.7(2.1)$ & $19.9(2.1)$ & $20.5(2.2)^{\mathrm{a}}$ \\
\hline Weekly number of alcoholic drinks & $20.5(17.3)$ & $16.3(13.6)$ & $20.4(17.1)$ & $23.1(19.2)^{\mathrm{a}}$ \\
\hline Number of hangovers per month & $2.7(2.4)$ & $2.1(1.7)$ & $2.7(2.6)$ & $2.9(2.4)$ \\
\hline Number of alcoholic drinks on heavy drinking session & $8.3(5.8)$ & $7.7(5.5)$ & $7.9(5.5)$ & $9.4(6.3)^{b}$ \\
\hline eBAC (\%) & $0.10(0.10)$ & $0.08(0.09)$ & $0.10(0.10)$ & $0.12(0.12)^{\mathrm{a}}$ \\
\hline Hangover severity & $23.5(13.9)$ & $27.5(16.5)$ & $23.9(13.4)$ & $20.9(12.5)^{\mathrm{a}}$ \\
\hline Hangover duration (h) & $18.4(3.9)$ & $17.5(4.4)$ & $18.3(3.7)$ & $19.2(3.9)^{\mathrm{a}}$ \\
\hline Total sleep time (h) & $6.5(2.1)$ & $3.4(0.9)$ & $6.0(0.7)$ & $9.0(1.4)^{\mathrm{a}-\mathrm{c}}$ \\
\hline
\end{tabular}

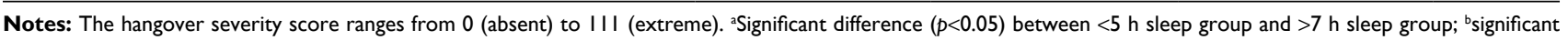
difference $(p<0.05)$ between $5-7 \mathrm{~h}$ sleep group and $>7 \mathrm{~h}$ sleep group; 'significant difference $(p<0.05)$ between $<5 \mathrm{~h}$ sleep group and $5-7 \mathrm{~h}$ sleep group.

Abbreviation: eBAC, estimated blood alcohol concentration. 
Table 2 Individual hangover symptoms and their severity

\begin{tabular}{|c|c|c|c|c|c|}
\hline Factor & Symptoms & Overall & $<5 \mathrm{~h}$ & 5-7 h & $>7 \mathrm{~h}$ \\
\hline \multirow[t]{5}{*}{ I Drowsiness } & Drowsiness & $5.8(2.9)$ & $5.8(3.1)$ & $5.8(2.8)$ & $5.6(3.0)$ \\
\hline & Fatigue & $6.5(2.6)$ & $7.1(2.7)$ & $6.6(2.4)^{\mathrm{c}}$ & $6.0(2.7)^{\mathrm{a}, \mathrm{b}}$ \\
\hline & Sleepiness & $5.6(3.1)$ & $6.4(3.0)$ & $5.5(3.0)^{c}$ & $5.3(3.1)^{\mathrm{a}}$ \\
\hline & Apathy & $4.2(3.3)$ & $4.3(3.5)$ & $4.1(3.2)$ & $4.3(3.4)$ \\
\hline & Weakness & $4.4(3.1)$ & $4.4(3.3)$ & $4.5(3.1)$ & $4.4(3.3)$ \\
\hline \multirow[t]{7}{*}{2 Cognitive problems } & Confusion & I.I (2.2) & $1.0(2.2)$ & I.I (2.2) & I.3 (2.3) \\
\hline & Disorientation & $\mathrm{I} .4(2.4)$ & I.3 (2.3) & $\mathrm{I} .4(2.4)$ & $1.6(2.5)$ \\
\hline & Increased reaction time & $3.4(2.8)$ & $3.4(2.9)$ & $3.3(2.7)$ & $3.6(2.8)$ \\
\hline & Reduced alertness & $3.7(2.8)$ & $3.5(3.0)$ & $3.7(2.7)$ & $3.9(2.8)$ \\
\hline & Concentration problems & $4.0(3.3)$ & $4.0(3.2)$ & $4.2(3.0)$ & $3.9(3.0)$ \\
\hline & Memory problems & $2.3(2.9)$ & $2.4(2.9)$ & $2.2(2.9)$ & $2.4(3.0)$ \\
\hline & Clumsiness & $2.2(2.7)$ & $2.1(2.6)$ & $2.2(2.7)$ & $2.2(2.7)$ \\
\hline \multirow[t]{8}{*}{3 Disturbed water balance } & Muscle pain & $1.5(2.5)$ & $1.7(2.6)$ & $1.5(2.6)$ & $1.2(2.5)$ \\
\hline & Dry mouth & $5.1(3.2)$ & $5.0(3.3)$ & $5.0(3.2)$ & $5.3(3.1)$ \\
\hline & Thirst & $5.9(3.0)$ & $6.2(2.9)$ & $5.7(3.1)$ & $6.1(2.8)$ \\
\hline & Tremor & $1.9(2.7)$ & $2.2(3.1)$ & $1.9(2.7)$ & $1.7(2.6)$ \\
\hline & Shivering & $1.5(2.5)$ & $2.0(2.8)$ & I.4 (2.4) & I.4 (2.4) \\
\hline & Sweating & $1.4(2.4)$ & $1.6(2.4)$ & $1.5(2.5)$ & $1.2(2.1)$ \\
\hline & Hot/cold flashes & $0.9(2.1)$ & $0.9(2.0)$ & $1.0(2.2)$ & $0.8(1.8)$ \\
\hline & Nystagmus & $0.6(1.6)$ & $0.7(1.5)$ & $0.7(1.8)$ & $0.6(1.6)$ \\
\hline \multirow[t]{4}{*}{4 Mood disturbances } & Depression & $0.8(2.0)$ & $1.0(2.4)$ & $0.7(1.8)$ & $0.9(2.0)$ \\
\hline & Anxiety & $0.3(1.1)$ & $0.4(1.4)$ & $0.3(1.1)$ & $0.3(1.1)$ \\
\hline & Anger & $0.3(1.1)$ & $0.4(\mathrm{I} .2)$ & $0.3(1.2)$ & $0.3(0.9)$ \\
\hline & Agitation & $2.0(2.6)$ & $1.6(2.2)$ & $2.1(2.6)$ & $2.1(2.6)$ \\
\hline \multirow[t]{4}{*}{5 Balance problems } & Balance problems & $1.6(2.5)$ & $1.5(2.4)$ & $1.5(2.5)$ & $1.7(2.5)$ \\
\hline & Dizziness & $2.0(2.8)$ & $2.5(3.2)$ & $2.0(2.7)$ & $1.9(2.7)$ \\
\hline & Vertigo & $1.9(2.8)$ & $2.3(3.1)$ & I.8 (2.7) & $1.9(2.7)$ \\
\hline & Tinnitus & $0.6(1.7)$ & $0.7(1.7)$ & $0.6(1.7)$ & $0.7(1.7)$ \\
\hline \multirow[t]{4}{*}{6 Gastrointestinal problems } & Nausea & $4.6(3.3)$ & $4.5(3.4)$ & $4.7(3.2)$ & $4.5(3.4)$ \\
\hline & Stomach pain & $2.0(2.7)$ & $2.3(3.0)$ & $2.1(2.8)$ & $1.6(2.5)$ \\
\hline & Gastrointestinal complaints & $2.2(2.9)$ & $2.3(3.1)$ & $2.3(2.9)$ & $2.0(2.9)$ \\
\hline & Gastritis & I.I (2.3) & $1.2(2.3)$ & $0.9(2.1)$ & I.3 (2.5) \\
\hline \multirow[t]{3}{*}{7 Respiratory and cardiovascular problems } & Palpitations & $0.7(1.9)$ & $0.9(2.1)$ & $0.6(1.8)$ & $0.8(1.8)$ \\
\hline & Pounding heart & $0.8(1.9)$ & I.I (2.3) & $0.7(1.9)^{c}$ & $0.7(1.7)$ \\
\hline & Respiratory problems & $0.4(1.3)$ & $0.8(2.0)$ & $0.3(1.3)^{c}$ & $0.2(0.9)^{\mathrm{a}}$ \\
\hline \multirow[t]{4}{*}{8 Impulsivity and blunted affect } & Restlessness & $1.6(2.6)$ & $1.9(3.0)$ & $1.6(2.5)$ & $1.6(2.6)$ \\
\hline & Blunted affect & $1.3(2.3)$ & $1.4(2.3)$ & $1.3(2.3)$ & $1.3(2.4)$ \\
\hline & Impulsivity & $0.9(2.1)$ & $1.3(2.5)$ & $0.8(2.0)$ & $0.9(2.1)$ \\
\hline & Loss of taste & I.I (2.I) & $1.6(2.7)$ & $0.9(1.9)$ & $1.2(2.2)$ \\
\hline \multirow[t]{4}{*}{9 Vomiting and feeling guilty } & Vomiting & $1.3(2.9)$ & $1.2(2.8)$ & $1.2(2.8)$ & $1.6(3.1)$ \\
\hline & Regret & $1.2(2.4)$ & $1.3(2.7)$ & I.I (2.2) & $1.3(2.5)$ \\
\hline & Guilt & I.I (2.2) & $1.5(2.7)$ & $0.9(1.9)$ & I.3 $(2.4)^{b}$ \\
\hline & Reduced appetite & $3.4(3.4)$ & $4.1(3.8)$ & $3.3(3.3)$ & $3.2(3.5)$ \\
\hline \multirow[t]{3}{*}{10 Headache } & Headache & $5.0(3.1)$ & $5.0(3.1)$ & $5.0(3.1)$ & $5.1(3.1)$ \\
\hline & Photosensitivity & $1.4(2.4)$ & I.3 (2.4) & $1.4(2.3)$ & $1.4(2.4)$ \\
\hline & Audio sensitivity & $1.4(2.3)$ & $1.3(2.6)$ & $1.4(2.2)$ & $1.5(2.4)$ \\
\hline I I Suicidal thoughts & Suicidal thoughts & $0.1(0.8)$ & $0.3(1.5)$ & $0.0(0.3)^{c}$ & $0.1(0.8)$ \\
\hline
\end{tabular}

Notes: Data represented as mean (SD). Symptoms are grouped according to the factor analysis. Data from Penning et al. ${ }^{6}$ aSignificant difference $(p<0.05)$ between $<5 \mathrm{~h}$ sleep group and $>7 \mathrm{~h}$ sleep group; 'bignificant difference $(p<0.05)$ between $5-7 \mathrm{~h}$ sleep group and $>7 \mathrm{~h}$ sleep group; ' significant difference $(p<0.05)$ between $<5 \mathrm{~h}$ sleep group and $5-7 \mathrm{~h}$ sleep group.

when compared to those who slept $<5$ hours. Compared to those who slept $<5$ hours, those who slept 5 to 7 hours had significantly less severe scores on fatigue $(p=0.012)$, sleepiness $(p=0.011)$, respiratory problems $(p=0.007)$, suicidal thoughts $(p=0.009)$, and pounding heart $(p=0.046)$.

\section{Discussion}

Our study confirms that drinkers with reduced TST report higher hangover severity scores after a night of heavy drinking. Subjects who slept longer than 7 hours consumed significantly more alcohol and reported an extended hangover duration. 
However, at the same time they reported having significantly less severe hangovers. The latter suggests that sleep has a positive effect on relieving alcohol hangover. TST was most strongly related to sleep-related individual hangover symptoms such as fatigue and sleepiness. Interestingly, short versus normal and long sleep did not significantly differ in severity scores of most commonly reported hangover symptoms. This observation demonstrates that some frequently reported hangover symptoms associated with moderate to high severity scores such as nausea and headache are independent of possible acute sleep deprivation after an evening of alcohol consumption.

Those with the shortest sleep durations consumed significantly less alcohol and had a much lower eBAC. From the present methodology, it is not possible to determine whether drinkers were aware of having limited time in bed, for example, due to a scheduled appointment next morning, and therefore reduced their alcohol intake relative to those who had the opportunity of unrestricted sleep. Future research should further investigate the impact of restricted versus unrestricted sleep opportunity on alcohol consumption and the severity and duration of the alcohol hangover.

A limitation of this study was that TST was the only sleep outcome assayed. No information was collected on sleep quality, nightly awakenings, time to sleep onset, or other potentially relevant parameters. Also, no objective measurements such as polysomnography or actigraphy were made to strengthen our observations. Future research using a prospective study design should implement the objective assessments. Applying a prospective study design would also eliminate possible recall bias, which is always an issue in retrospective survey research. It would also be of interest to include psychometric assessments in future studies. Although it is known that cognitive and psychomotor functioning and daily activities such as driving a car may be impaired during alcohol hangover, ${ }^{8,9}$ the impact of variations in TST on performance during the hangover state has not received adequate research attention. The observation that TST is significantly related to hangover severity does however predict that variations in TST may also affect daily activities during the hangover state such as driving a car or job performance. Finally, it should be kept in mind that the direction of the association between total sleep time and alcohol consumption and hangover severity is unknown. From the current survey data, cause and effect cannot be determined.

\section{Conclusion}

The current data confirm that an evening of heavy drinking often occurs at the expense of total sleep time. Reduced sleep time is associated with more severe hangovers.

\section{Acknowledgment}

An abstract containing interim findings of this paper was presented as a poster at the 2014 British Association for Psychopharmacology meeting. The poster's abstract was published in Journal of Psychopharmacology 2014, 28 (supplement to \#8): A19.

\section{Disclosure}

Joris C Verster has received grants/research support from the Dutch Ministry of Infrastructure and the Environment, Janssen, Nutricia, Red Bull, and Takeda, and has acted as a consultant for the Canadian Beverage Association, Centraal Bureau Drogisterijbedrijven, Coleman Frost, Danone, Deenox, Eisai, Janssen, Jazz, Purdue, Red Bull, Sanofi-Aventis, Sen-Jam Pharmaceutical, Sepracor, Takeda, Transcept, Trimbos Institute, and Vital Beverages. Thomas Roth has received grants/research support from Aventis, Cephalon, GlaxoSmithKline, Neurocrine, Pfizer, Sanofi, Schering-Plough, Sepracor, Somaxon, Syrex, Takeda, TransOral, Wyeth, and XenoPort and has acted as a consultant for Abbott, Acadia, Acoglix, Actelion, Alchemers, Alza, Ancil, Arena, AstraZeneca, Aventis, AVER, BMS, BTG, Cephalon, Cypress, Dove, Elan, Eli Lilly, Evotec, Forest, Glaxo Smith Kline, Hypnion, Impax, Intec, Intra-Cellular, Jazz, Johnson \& Johnson, King, Lundbeck, McNeil, MediciNova, Merck, Neurim, Neurocrine, Neurogen, Novartis, Orexo, Organon, Prestwick, Procter \& Gamble, Pfizer, Purdue, Resteva, Roche, The other authors report no conflicts of interest in this work.

\section{References}

1. Van Schrojenstein Lantman M, Mackus M van de Loo AJAE, Verster JC. Development of a definition for the alcohol hangover: consumer descriptions and expert consensus. Curr Drug Abuse Rev. 2016;9:148-154.

2. Finnigan F, Hammersley R, Cooper T. An examination of next-day hangover effects after a $100 \mathrm{mg} / 100 \mathrm{ml}$ dose of alcohol in heavy social drinkers. Addiction. 1998;93:1829-1938.

3. McKinney A, Coyle K. Alcohol hangover effects on measures of affect the morning after a normal night's drinking. Alcohol Alcohol. 2006;41:54-60.

4. Rohsenow DJ, Howland J, Minsky SJ, Arnedt JT. Effects of heavy drinking by maritime academy cadets on hangover, perceived sleep, and next-day ship power plant operation. J Stud Alcohol. 2006; 67:406-415.

5. Rohsenow DJ, Howland J, Arnedt JT, et al. Intoxication with bourbon versus vodka: effects on hangover, sleep, and next-day neurocognitive performance in young adults. Alcohol Clin Exp Res. 2010;34:509-18.

6. Penning R, McKinney A, Verster JC. Alcohol hangover symptoms and their contribution to overall hangover severity. Alcohol Alcohol. 2012; 47:248-252.

7. Watson PE, Watson ID, Batt RD. Prediction of blood alcohol concentrations in human subjects. Updating the Widmark Equation. J Stud Alcohol. 1981;42:547-556.

8. Ling J, Stephens R, Heffernan TM. Cognitive and psychomotor performance during alcohol hangover. Curr Drug Abuse Rev. 2010;3:80-87.

9. Verster JC, Bervoets AC, de Klerk S, et al. Effects of alcohol hangover on simulated highway driving performance. Psychopharmacology (Berl). 2014;231:2999-3008. 


\section{Publish your work in this journal}

Nature and Science of Sleep is an international, peer-reviewed, open access journal covering all aspects of sleep science and sleep medicine, including the neurophysiology and functions of sleep, the genetics of sleep, sleep and society, biological rhythms, dreaming, sleep disorders and therapy, and strategies to optimize healthy sleep. The manuscript management system is completely online and includes a very quick and fair peer-review system, which is all easy to use. Visit http://www dovepress.com/testimonials.php to read real quotes from published authors.

Submit your manuscript here: https://www.dovepress.com/nature-and-science-of-sleep-journal 